\section{PWE-128 SURVEILLANCE OF HEPATOCELLULAR CARCINOMA - CONSISTENT OR CONFUSED?}

${ }^{1}$ B Hudson*, ${ }^{2}$ L Lee, ${ }^{2} \mathrm{JD}$ Maltby. ${ }^{1}$ Department of Hepatology, University Hospitals Bristol NHS Trust, Bristol, UK; ${ }^{2}$ Gastroenterology, Royal United Hospital, Bath, UK

\subsection{6/gutjnl-2014-307263.388}

Introduction BSG guidelines for diagnosis and treatment of hepatocellular carcinoma (2003) advocate 6 monthly surveillance of high risk cirrhotic patients with abdominal ultrasonography and alfa-feto protein estimation.

An audit of cirrhotic patients managed at the Royal United Hospital demonstrated poor compliance with BSG guidelines, with only $24.1 \%$ of eligible patients receiving regular 6 monthly surveillance over an 18 month period. Compliance was particularly poor amongst viral hepatitis patients who often failed to attend appointments.

The current work explores whether the difficulties and inconsistencies noted on a local level are representative of wider practice, and considers ways in which barriers to optimum practice could be overcome.

Methods Issues raised from a local audit (see above) informed design of an electronic questionnaire which assessed policy, clinician opinion, and response to various clinical scenarios. This was distributed to Gastroenterology/Hepatology consultants and STRs in the South West and Wales.

Results 81 responses were received from 16 NHS trusts across the South West and Wales (42\% response rate). $41 \%$ of respondents were consultants (59\% gastroenterologists $/ 41 \%$ hepatologists). $65.3 \%$ of respondents were familiar with BSG guidelines, however only $21.8 \%$ used them within their institution. $33 \%$ of respondents did not know which guidelines their department used.

Widespread variation was noted in response to clinical scenarios. Whereas there was general agreement that 6 monthly surveillance should be afforded to patients with cirrhosis secondary to haemochromatosis and alcohol when abstinent (even amongst females which is not suggested in BSG guidelines), opinion was divided in respect to patients who continued to drink, and in those with non-cirrhotic chronic hepatitis B (47\% would offer surveillance, $36 \%$ would not).

Poor patient compliance and insufficient resources and expertise to co-ordinate surveillance programmes were cited as the main barriers to successful surveillance. $86 \%$ of respondents felt HCC surveillance could be improved within their institution, and 38\% thought HCC surveillance programmes should be further extended given recent developments in palliative management.

Conclusion Findings from this study would, if representative of wider practice, suggest considerable variations in HCC surveillance accross the UK currently exist. Low levels of compliance with and awareness of BSG guidelines were demonstrated. Opinion regarding optimum surveillance of certain pateint groups (e. g., non-cirrhotic viral hepatitis and alcoholic cirrhosis in females) was generally at odds with guidelines. Updating guidelines to account for recent changes in HCC management may help to achieve nationally consistent high quality HCC surveillance. Strategies for improving local HCC surveillance are discussed. Disclosure of Interest None Declared.

\section{PWE-129 TRENDS IN VARICEAL BLEEDING: A SINGLE CENTRE EXPERIENCE FROM 2006-2013}

${ }^{1} \mathrm{C}$ Grant*, 'D Kemp, ${ }^{2} \mathrm{M}$ Beattie, 'A Austin. 'Department of Gastroenterology, Royal Derby Hospital, Derby; ${ }^{2}$ Faculty of Medicine, University of Nottingham, Nottingham, UK

10.1136/gutjnl-2014-307263.389
Introduction Over the last decade, the numbers of patients presenting with chronic liver disease has risen. During this period the approach to the treatment of variceal bleeding has undergone important changes both internationally (adoption of early TIPSS in high risk cases), and locally with the development of a $24 \mathrm{~h}$ endoscopy service (2006), movement to single site hospital with enlarged intensive care capacity (2009), adoption of the Danis ${ }^{\mathrm{TM}}$ stent (2009) and a shift to carvedilol as the primary agent for prophylaxis (2013). We reviewed all episodes of variceal bleeding in the last 8 years to describe patient outcomes.

Methods All episodes of bleeding from oesophageal varices managed in the Liver Unit at Royal Derby Hospital from 2005 to mid 2013 were identified from clinical coding data - population served approx. 650,000. A retrospective review of the patient records identified the aetiology and severity of liver disease, morbidity, mortality, endoscopy findings and episodes of rebleeding.

Results Each year between 17 and 31 patients presented with variceal bleeding. 5 day mortality fluctuated between 3-22\% whereas 30 day mortality fell steadily from a peak in 2006 of $41 \%$ to $5 \%$ in 2012 (Figure 1). The reduction in mortality was in Child's B/C cirrhosis. Interestingly, the proportion of episodes in Child's A cirrhosis increased from 2009 onwards (7\% of all bleeding episodes in 2009 to above $30 \%$ in 2013). 30 day mortality rates for Child's A did not improve but remained lower than for those with Child's B/C cirrhosis (mean 9.8\% compared to $22.8 \%$ (2009-2013)). From 2007, there was a fall in frequency of rebleeding from $35 \%$ to below $10 \%$ in 2013 . Only 3 high risk patients underwent an early TIPSS procedure, all after 2012.

Conclusion Variceal bleeding rates have remained surprisingly constant over 8 years despite the rise in admissions with chronic liver disease. Outcomes for acute variceal bleeding have improved which is likely the result of several organisational changes. Notably, rebleeding rates and 30 day mortality decreased even before the adoption of early TIPSS.

Disclosure of Interest None Declared.

\section{Mortality Rates for Variceal Bleeding}



Abstract PWE-129 Figure 1

\section{PWE-130 PHENOTYPE AND LOCALISATION OF LIVER INFILTRATING B CELL SUBSETS IN AUTOIMMUNE AND INFLAMMATORY LIVER DISEASES}

D Geh* ${ }^{*}$ H Jeffery, DH Adams, YH Oo. Centre for Liver Research and NIHR BRU, University of Birmingham, Birmingham, UK

10.1136/gutjnl-2014-307263.390 